\title{
Métodos geoestatísticos na modelagem espacial do diâmetro médio do cristal da goethita
}

\author{
João F. da Silva Junior ${ }^{1}$, Gener T. Pereira ${ }^{1}$, Livia A. Camargo ${ }^{1}$ \& José Marques Júnior ${ }^{1}$
}

\section{RESUMO}

Uma das necessidades da agricultura de precisão é avaliar a qualidade dos mapas dos atributos dos solos. Neste sentido, o presente trabalho objetivou avaliar o desempenho dos métodos geoestatísticos: krigagem ordinária e simulação sequencial gaussiana na predição espacial do diâmetro médio do cristal da goethita com 121 pontos amostrados em uma malha de 1 ha com espaçamentos regulares de 10 em $10 \mathrm{~m}$. Após a análise textural e da concentração dos óxidos de ferro, calcularam-se os valores do diâmetro médio do cristal da goethita os quais foram analisados pela estatística descritiva e geoestatística; em seguida, foram utilizadas a krigagem ordinária e a simulação sequencial gaussiana. Com os resultados avaliou-se qual foi o método mais fiel para reproduzir as estatísticas, a função de densidade de probabilidade acumulada condicional e a estatística epsilon $\varepsilon_{y}$ da amostra. As estimativas E-Type foram semelhantes à krigagem ordinária devido à minimização da variância. No entanto, a krigagem deixa de apresentar, em locais específicos, o grau de cristalinidade da goethita enquanto o mapa E-Type indicou que a simulação sequencial gaussiana deve ser utilizada ao invés de mapas de krigagem. Os mapas E-type devem ser preferíveis por apresentar melhor desempenho na modelagem.

Palavras-chave: agricultura de precisão, krigagem ordinária, simulação sequencial gaussiana, pedometria

\section{Geostatistical methods in spatial modelling of the middle diameter of the goethite crystal}

\begin{abstract}
In precision agriculture it is necessary to assess the quality of the maps of soil attributes. This paper aimed to evaluate the performance of geostatistical methods: ordinary kriging and sequential Gaussian simulation on spatial prediction of mean diameter of the goethite crystal in a regular grid with 121 points collected in a grid of 1 ha with regular spacing of 10 to $10 \mathrm{~m}$. After the particle size and concentration analysis of iron oxides the values of the mean diameter of the goethite crystal were calculated. These were analysed by descriptive statistics and geostatistics, ordinary kriging and sequential Gaussian simulation was used for production of maps. With the results which evaluated the method is more faithful to make statistics, the cumulative probability density function conditional epsilon statistics $\varepsilon_{\mathrm{y}}$. E-Type estimates were similar to ordinary kriging, due to the minimization of the variance. However, the ordinary kriging did not perform well in specific locations for crystallinity of goethite, while the E-Type map by sequential Gaussian simulation should be used instead of kriging maps. The E-type maps should be prefered as they present better performance in modeling.
\end{abstract}

Key words: precision agriculture, ordinary kriging, sequential gaussian simulation, pedometrics 


\section{INTRODUÇÃO}

Cada vez mais os pesquisadores têm presenciado a importância em estudar a variabilidade espacial dos atributos do solo. Desde as primeiras aplicações da geoestatística em solos nos anos 80 (Burgess \& Webster, 1980), os estudiosos da ciência do solo (pedólogos) já estão convencidos de que os solos possuem variabilidade natural, pelo seu material de origem, além da variabilidade encontrada no sentido vertical, e também no sentido horizontal (Zanão Júnior et al., 2010). Nesses paradigmas vários estudos do solo têm sua atenção direcionada no sentido de se estudar a variabilidade espacial, caracterizando-a, o que é possível em virtude do avanço da informática, que propiciou a aplicação prática da teoria das variáveis regionalizadas chamada geoestatística (Matheron, 1963).

$\mathrm{Na}$ literatura existem vários trabalhos sobre variabilidade espacial de atributos do solo (Camargo et al., 2008; Oliveira Júnior et al., 2011 e Silva Júnior et al., 2012a,b) em que a maioria utiliza a krigagem ordinária $(\mathrm{KO})$ para a interpolação na confecção de mapas de variabilidade espacial, por ser considerado um interpolador local ótimo, uma vez que seu princípio é a estimativa ótima não enviesada nos pontos não amostrados usando as propriedades estruturais dos semivariogramas com variância mínima da sua estimativa e sem tendência (Isaaks \& Srivastava, 1989). Ela é uma média ponderada pelos pesos $\lambda_{i}$ da sua vizinhança, dentro de um raio de influência de correlação espacial modelada pelo semivariograma porém a KO representa apenas uma variabilidade média dos atributos da área de estudo modelada pelo semivariograma (Silva Júnior et al., 2012a). Ela não fornece acesso à incerteza e a suavização de mapas pela krigagem ordinária pode aumentar ainda mais o erro no manejo localizado, erro total na modelagem, juntamente com erros de amostragem e de análises laboratoriais, principalmente em pequenas escalas.

Deste modo outra alternativa é a simulação sequencial gaussiana (SSG) que é baseada em uma pressuposição multigaussiana do modelo de uma função aleatória (Bourennane et al., 2010). Ela possui a vantagem de integrar, de forma mais simples, vários atributos em um único modelo visando à apresentação da estatística dos dados amostrais (Ortiz et al., 2004).

Na SSG muitos mapas, igualmente prováveis da distribuição do atributo em estudo, são produzidos de acordo o mesmo modelo da correlação espacial tal como requerido para a krigagem. Neste sentido, o presente trabalho objetivou analisar o desempenho de métodos geoestatísticos: krigagem ordinária e simulação sequencial gaussiana na reprodução da variabilidade espacial do diâmetro médio do cristal da goethita.

\section{Material e Métodos}

A área de estudo está situada na Fazenda Boa Vista, pertencente à Usina São Domingos Açúcar e Álcool, no município de Tabapuã, região nordeste de São Paulo, nas coordenadas geográficas $21^{\circ} 05^{\prime} 57,11^{\prime}$ " de latitude sul e $49^{\circ}$ $01^{\prime} 02,08 "$ de longitude oeste. O solo foi classificado como Argissolo Vermelho-Amarelo eutrófico textura média/argilosa
(EMBRAPA, 2006). O clima da região foi classificado pelo método de Köppen, como tropical quente úmido, tipo Aw.

Instalou-se um malha amostral de dimensão $100 \times 100 \mathrm{~m}$ delimitada em uma área com espaçamento regular de $10 \times 10$ $\mathrm{m}$; os pontos de cruzamento deste espaçamento determinaram os pontos de coleta das amostras na profundidade de $0,0-0,20$ $\mathrm{m}$, no total de 113 pontos amostrais georreferenciados por meio de um receptor de sistema de posicionamento global (GPS) em cada ponto da malha, conforme Figura 1.

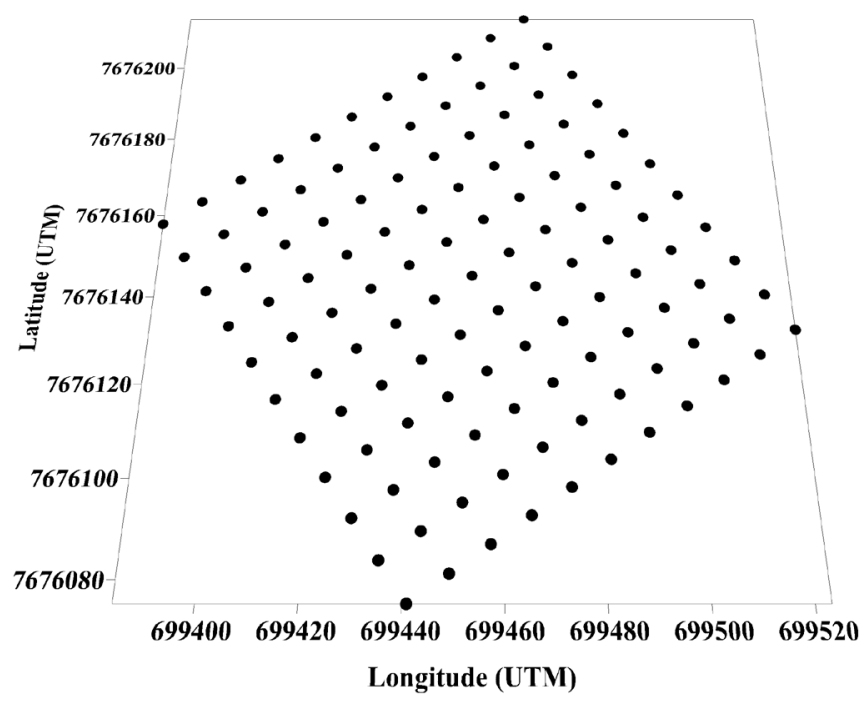

Figura 1. Pontos de amostragem $(\bullet)$ em malha regular

As amostras de solo foram submetidas à análise granulométrica utilizando-se uma solução de $\mathrm{NaOH} 0,5 \mathrm{~N}$; em seguida à agitação mecânica, por 10 min, para dispersão das partículas; após este tratamento prévio a fração areia foi retirada em peneira de $0,05 \mathrm{~mm}$.

A separação da fração silte e da areia ocorreu por centrifugação (1600 rpm). A suspensão de argila foi floculada com $\mathrm{HCl}$ concentrada e centrifugada (2000 rpm por $2 \mathrm{~min}$ ). A caracterização da goethita (Gt) por meio da difração de raios-X (DRX), foi feita após tratamento da fração argila com $\mathrm{NaOH} 5$ mol L ${ }^{-1}$ (100 mL solução $1 \mathrm{~g}^{-1}$ de argila), para concentração dos óxidos de ferro de conformidade com o método modificado por Kämpf \& Schwertmann (1982).

Para evitar as mudanças na substituição em alumínio e cristalinidade da Gt foram adicionados $10 \%$ em peso de sílica gel moída para a manutenção de concentração mínima de ácido silícico na solução de $\mathrm{NaOH} 5 \mathrm{~mol} \mathrm{~L}^{-1}$. A fim de que a leitura dos difratogramas não fosse dificultada pela sodalita, as amostras foram lavadas com solução de $\mathrm{HCl} 0,5 \mathrm{~mol} \mathrm{~L}^{-1}(100$ $\mathrm{mL}$ de solução $1 \mathrm{~g}^{-1}$ de argila) em agitação por $4 \mathrm{~h}$.

Visando corrigir os desvios no posicionamento (d) dos reflexos estudados foram acrescentados, às amostras, $10 \% \mathrm{em}$ peso de cloreto de sódio moído e peneirado em malha $0,10 \mathrm{~mm}$, antes de serem difratados. A difração de raios X foi realizada com as amostras preparadas pelo método do pó, em aparelho HGZ equipado com cátodo de cobalto e filtro de ferro e radiação $\mathrm{K} \alpha$, com uma corrente de $20 \mathrm{~mA}$ e tensão de $30 \mathrm{kV}$ para difração da Gt. O diâmetro médio do cristal (DMC) da Gt foi calculado a partir da largura meia altura (LMA) e da posição dos reflexos da Gt no plano (110), conforme Camargo et al. (2008). 
Inicialmente, os valores do DMC foram analisados por meio da estatística descritiva mínimo, máximo, média, desvio padrão, $1^{\circ}$ quartil (Q1), $3^{\circ}$ quartil (Q3) coeficientes de variação, assimetria e curtose. Os valores de $\mathrm{CV}$ foram classificados como: baixa variabilidade $(\mathrm{CV}<12 \%)$, média variabilidade $(12 \% \mathrm{CV}<62 \%)$ e alta variabilidade $(\mathrm{CV}>62 \%)$, Warrick \& Nielsen (1980).

A análise da dependência espacial do DMC da Gt foi avaliada por meio de ajustes de modelos matemáticos aos semivariograma experimentais e a escolha do melhor modelo ajustado foi baseada (1) na menor soma do quadrado do resíduo; (2) no maior coeficiente de determinação $\left(\mathrm{R}^{2}\right)$ e com base na pressuposição de estacionariedade da hipótese intrínseca (Matheron, 1963) estimado por meio da expressão:

$$
\gamma(\mathrm{h})=\frac{1}{2 \mathrm{~N}(\mathrm{~h})} \sum_{\mathrm{i}=1}^{\mathrm{N}(\mathrm{h})}\left[\mathrm{Z}\left(\mathrm{x}_{\mathrm{i}}\right)-\mathrm{Z}\left(\mathrm{x}_{\mathrm{i}}+\mathrm{h}\right)\right]^{2}
$$

sendo:

$\mathrm{N}(\mathrm{h})$ - número de pares experimentais de observações

$\mathrm{Z}\left(\mathrm{x}_{\mathrm{i}}\right)$ e $\mathrm{Z}\left(\mathrm{x}_{\mathrm{i}}+\mathrm{h}\right)$ separados por uma distância $\mathrm{h}$

O semivariograma é representado pelo gráfico de $\gamma(\mathrm{h})$ versus $h$.

A partir do ajuste de um modelo matemático aos valores calculados de $\gamma(\mathrm{h})$, são estimados os parâmetros do modelo teórico do semivariograma (o efeito pepita, $\mathrm{C}_{0}$; patamar, $\mathrm{C}_{0}+$ $\mathrm{C}_{1}$ e o alcance, a). Foram testados os seguintes modelos: (a) esférico; (b) exponencial; (c) gaussiano e linear (d).

Após a obtenção do semivariograma experimental foi ajustado um modelo teórico permissível que melhor o representa; em seguida foram estimados, com base nos coeficientes do semivariograma teórico, os valores do DMC da Gt em determinada posição geográfica não amostrada da área de estudo, por meio da krigagem ordinária $(\mathrm{KO})$ pela seguinte expressão:

$$
z\left(x_{0}\right)=\sum_{i=1}^{N} \lambda_{i} z\left(x_{i}\right)
$$

sendo:

$\mathrm{z}\left(\mathrm{x}_{0}\right)$ - estimativa de krigagem no ponto $\mathrm{x}_{0}$

$z\left(x_{i}\right)$ - valores medidos em $x_{i}, i=1,2, \ldots, N$

$\lambda_{i} \quad$ - pesos da krigagem calculados com base no semivariograma ajustado são atribuídos aos valores vizinhos $\mathrm{z}\left(\mathrm{x}_{\mathrm{i}}\right)$ para estimar $\mathrm{z}\left(\mathrm{x}_{0}\right)$

Com vista à realização da simulação sequencial gaussiana (SSG) seguiram-se as etapas: (1) Transformação da distribuição experimental em uma distribuição gaussiana padronizada; (2) Ajuste de um modelo de semivariograma a partir dos dados gaussianos da etapa 1; (3) Definição de um caminho aleatório na área sendo que cada ponto seja visitado apenas uma vez; (4) Construção de uma função de densidade de probabilidade acumulada condicional (F.D.P.A.C) no local $x_{i}$ via KO, condicionado à informação da vizinhança de $\mathrm{x}_{\mathrm{i}}$; (5) Seleção aleatória de um valor da F.D.P.A.C que passará a representar este local e inclusão deste valor no banco de dados como informação condicional adicional utilizando-se as demais visitas; (6) Ir ao próximo ponto estabelecido no caminho aleatório e repetir os passos anteriores; (7) Repetição das etapas de 4-5 até que os $\mathrm{N}$ pontos da malha refinada tenham sido simulados; (8) Transformação de volta do valor simulado para a escala original dos dados amostrais (ao finalizar esta etapa é gerada uma realização ou imagem estocástica). Para gerar outra realização deve-se retornar à etapa 3 e repetir as etapas até a 8 .

A partir das 200 realizações foi gerado o mapa médio de distribuição espacial do DMC da Gt por meio do cálculo da média pontual de todas as realizações fornecendo as estimativas denominadas E-type (Deutsch \& Journel, 1998), conforme ilustração da simulação estocástica na Figura 2. Para o estudo das realizações foram sorteadas, aleatoriamente, as realizações individuais: $1 ; 37 ; 128$ e 200.

A acurácia da reprodução do semivariograma pelos métodos geoestatísticos foi avaliada pela estatística epsilon $\varepsilon_{\mathrm{y}}$ (Goovaerts, 2000).

$$
\varepsilon_{\mathrm{y}}=\sum_{\mathrm{s}=1}^{\mathrm{s}} \frac{\left[\mathrm{y}\left(\mathrm{h}_{\mathrm{s}}\right)-\mathrm{y}\left(\mathrm{h}_{\mathrm{s}}\right)\right]^{2}}{\left[\mathrm{y}\left(\mathrm{h}_{\mathrm{s}}\right)\right]^{2}}
$$

em que: $\mathrm{S}$ é o número de intervalos de distância $\mathrm{h}$ utilizados para a construção do semivariograma; $y\left(h_{s}\right)$, a semivariância na distância $h_{s}$, calculada a partir dos valores estimados pelo método de interpolação e/ou simulação e $\mathrm{y}\left(\mathrm{h}_{\mathrm{s}}\right) \mathrm{o}$ valor da semivariância do modelo ajustado na distância $h_{\mathrm{s}}$. Devido à divisão pelo quadrado, mais peso é dado à reprodução do modelo de semivariograma próximo à origem, ou seja, menores distâncias $\mathrm{h}$, sendo esta região a mais relevante aos cálculos de interpolação.

Os menores valores de $\varepsilon_{\mathrm{y}}$ indicam boa reprodução do semivariograma de referência por parte do semivariograma estimado pelos métodos de interpolação e/ou simulação (Bourennane et al., 2007).
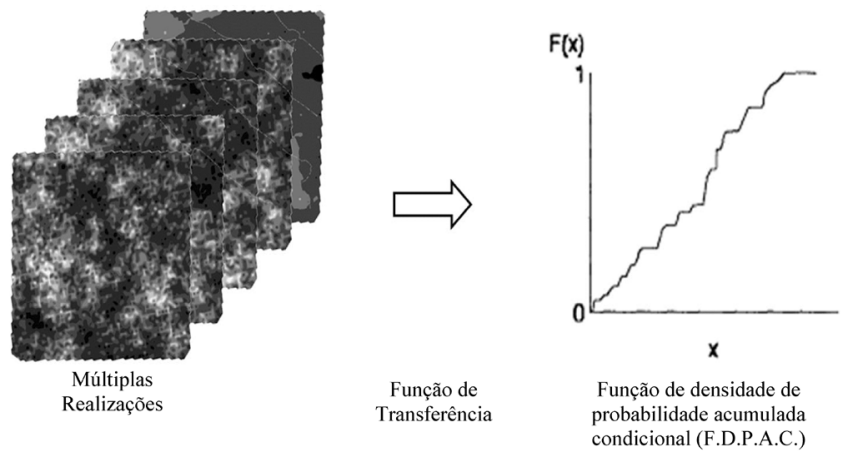

Figura 2. Ilustração da simulação estocástica adaptado de Journel (1989)

\section{Resultados E Discussão}

Os resultados das estatísticas descritivas das realizações individuais $\left(1^{\mathrm{a}}, 37^{\mathrm{a}}, 128^{\mathrm{a}}\right.$ e $\left.200^{\mathrm{a}}\right)$ da SSG e E-type (Tabela 1) preservaram bem a faixa de variabilidade observando-se que dentre os valores obtidos referentes ao $1^{\circ}$ Quartil $\left(Q_{1}\right)$, mediana, $2^{\circ}$ Quartil $\left(\mathrm{Q}_{2}\right)$, desvio padrão (D.P), coeficientes de variação $\mathrm{CV} \%$, assimetria e curtose do $\mathrm{DMC}$ da Gt são mais 
Tabela 1. Estatísticas descritivas das amostras, das simulações SSG1a , SSG37a , SSG128a , SSG200a , E-Type e KO do diâmetro médio do cristal (DMC) da Gt

\begin{tabular}{|c|c|c|c|c|c|c|c|}
\hline Estatística descritiva & Amostra & SSG1 ${ }^{a}$ & SSG37 $^{\mathbf{a}}$ & SSG128 & SSG200 ${ }^{a}$ & E-Type & KO \\
\hline Número de pontos & 121 & 4.828 & 4.828 & 4.828 & 4.828 & 4.828 & 4.928 \\
\hline Média & 31,77 & 30,07 & 29,93 & 30,24 & 30,33 & 30,39 & 31,31 \\
\hline Mediana & 28,50 & 28,49 & 28,47 & 28,49 & 28,50 & 29,39 & 30,38 \\
\hline $\mathrm{DP}^{(1)}$ & 11,00 & 8,70 & 8,98 & 9,29 & 9,10 & 4,89 & 3,94 \\
\hline Mínimo & 15,06 & 15,06 & 15,06 & 15,06 & 15,06 & 15,06 & 20,62 \\
\hline$Q 1^{(2)}$ & 23,32 & 23,32 & 23,32 & 23,32 & 23,32 & 26,70 & 28,20 \\
\hline $\mathrm{Q3}^{(3)}$ & 36,78 & 36,77 & 36,77 & 36,78 & 36,78 & 33,64 & 34,12 \\
\hline Máximo & 86,91 & 61,71 & 61,71 & 61,71 & 61,71 & 61,71 & 52,53 \\
\hline $\mathrm{CV}^{(4)}$ & 34,64 & 28,90 & 30,01 & 30,73 & 30,01 & 16,10 & 12,58 \\
\hline Assimetria & 2,02 & 1,27 & 1,20 & 1,35 & 1,24 & 0,89 & 0,82 \\
\hline Curtose & 5,94 & 1,61 & 1,31 & 1,69 & 1,34 & 1,08 & 0,69 \\
\hline
\end{tabular}

(1) DP - Desvio padrão; (2) $Q 1-1^{0}$ quartil; (3) $Q 3-3^{0}$ quartil; (4) $\mathrm{CV}$ - Coeficiente de variação

semelhantes ao conjunto amostral do $\mathrm{DMC}$ em relação aos mapas de $\mathrm{KO}$ enquanto a $\mathrm{KO}$ reproduz apenas a variabilidade média da amostra do DMC da Gt visto que, com o valor mais próximo da média da amostra, de 31,77 e $31,31 \mathrm{~nm}$ para a $\mathrm{KO}$, respectivamente, os coeficientes de assimetria e curtose do DMC $(2,02$ e 5,94).

Os valores de $\mathrm{CV} \%$ das realizações individuais, estimativas E-type, KO e da amostra são classificados como variabilidade média e conforme Warrick \& Nielsen (1980), que corroboram com Oliveira Júnior et al. (2011) este fato revela o efeito maior da minimização da variância da estimativa (suavização) da $\mathrm{KO}$ em relação à E-type, que é característica deste método de interpolação (Isaaks \& Srivastava, 1989). Resultados semelhantes foram encontrados por Lookman et al. (1995) utilizando a $\mathrm{KO}$ para interpolação espacial de atributos da capacidade de sorção de fosfato em óxido de Fe e Al.

O modelo com o melhor ajuste foi o exponencial (Tabela 2), apresentando um índice de dependência espacial moderada, de acordo com Cambardella et al. (1994). O valor de alcance foi de $27,26 \mathrm{~m}$, este valor pode estar relacionado ao fato de que a malha amostral está localizada em forma convexa do relevo a qual é considerada uma das causas da variabilidade dos atributos dos solos (Montanari et al., 2010; Silva Júnior et al., 2012a).

A KO superestima os valores mínimos e subestima os valores máximos buscando reproduzir os valores médios do atributo enquanto a SSG busca reproduzir o realismo da variabilidade do atributo. Resultados semelhantes foram encontrados por Teixeira et al. (2011) avaliando métodos geoestatísticos. Esta afirmação pode ser constatada na Figura $3 \mathrm{em}$ que a função densidade de probabilidade condicional (FDPAC) de referência do DMC da Gt é reproduzida pelas FDPAC das realizações estocásticas condicionais.

Assim, os mapas mais realistas foram conseguidos com simulação estocástica condicionada pela SSG e estão de acordo com Rezaee et al. (2011), em que a KO tem, como objetivo uma estimativa local melhor sem preocupação com o efeito da suavização da variância nem a reprodução da estatística.

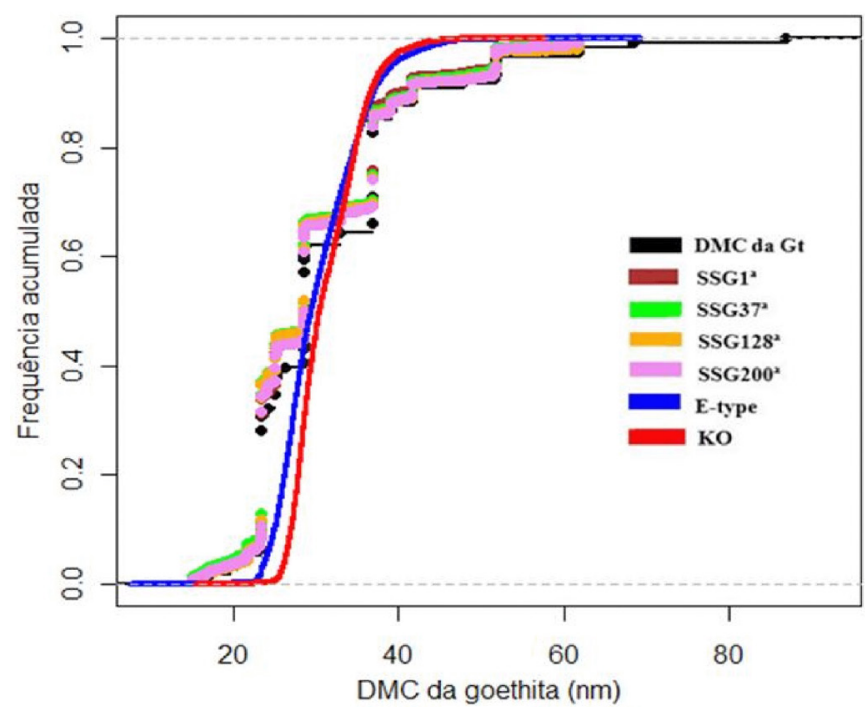

Figura 3. Funções de densidade de probabilidade acumulada condicional do diâmetro médio do cristal da goethita (nm): conjunto amostral (DMC da Gt) vs realizações da SSG $\left(1^{\mathrm{a}} ; 37^{\mathrm{a}} ; 128^{\mathrm{a}}\right.$ e $\left.200^{\mathrm{a}}\right)$, média das 200 realizações (E-type) e krigagem ordinária (KO)

É importante destacar o poder da SSG em representar locais específicos onde a $\mathrm{KO}$ está suavizando (mascarando) os valores extremos (mínimo e máximo) do DMC da Gt, conforme retângulo pontilhado nas Figuras 4E e 4F, os quais corroboram com os de Lookman et al. (1995) estudando a variabilidade espacial da capacidade de sorção de fosfato em óxido de ferro e alumínio, Teixeira et al. (2011) estimando a emissão de $\mathrm{CO}_{2}$ com diferentes interpoladores.

As realizações individuais são semelhantes porém não idênticas; apresentam mesmo padrão espacial do DMC da Gt. A subestimação e a superestimação da KO e da média das 200 realizações da SSG apresentada na Tabela 1 se refletem em mapas suavizados (Figuras 4E e 4F).

O mapa originado por meio da $\mathrm{KO}$ do DMC deixa de apresentar, em locais específicos, esta característica do grau de cristalinidade da Gt, o que não ocorre no mapa E-Type,

Tabela 2. Descrição dos parâmetros do modelo teórico do semivariograma do diâmetro médio do cristal (DMC) da goethita

\begin{tabular}{cccccccc}
\hline Atributo & Modelo & $\mathbf{C}_{0}{ }^{(1)}$ & $\mathbf{C}_{0}+\mathbf{C}_{1}^{(2)}$ & $\mathbf{a}^{(3)}$ & $\mathbf{I D E}^{(4)}$ & $\mathbf{R}^{(2)}$ & $\mathbf{S Q R}^{(\mathbf{6})}$ \\
$\mathrm{DMC}(\mathrm{Gt})$ & Exponencial & 27,00 & 85,03 & 27,26 & 31,75 & 0,86 & 79,00 \\
\hline
\end{tabular}

(1) $\mathrm{C}_{0}-$ Efeito pepita; (2) $\mathrm{C}_{0}+\mathrm{C}_{1}-$ Patamar; ( ${ }^{(3)} \mathrm{a}-$ Alcance $(\mathrm{m}) ;{ }^{(4)}$ IDE: $\mathrm{C}_{0} /\left(\mathrm{C}_{0}+\mathrm{C}_{1}\right) \times 100$ - Índice de dependência espacial (\%); ${ }^{(5)} \mathrm{R}^{2}-$ Coeficiente de determinação; ${ }^{(6)}$ SQR - Soma do quadrado do resíduo 
A. SSG $1^{\text {a }}$

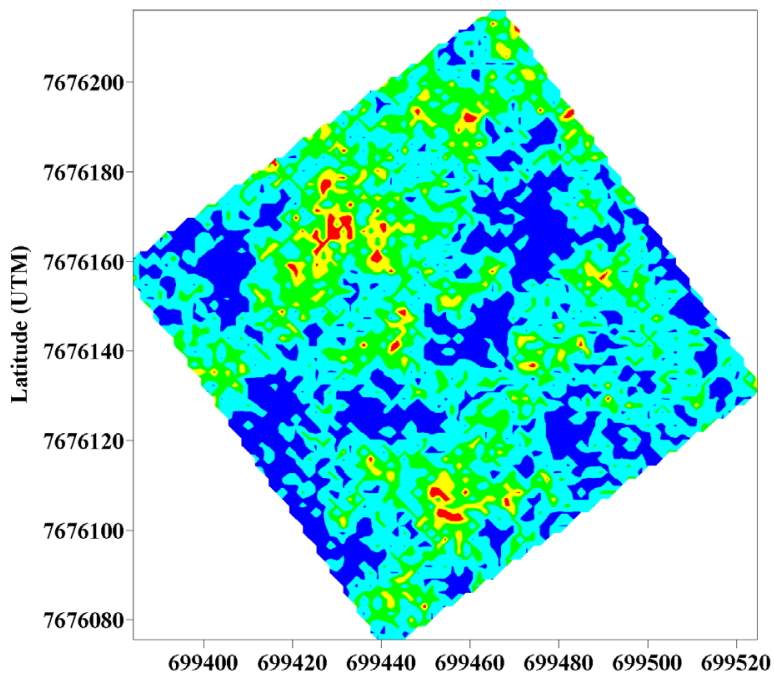

C. $\mathrm{SSG} 128$

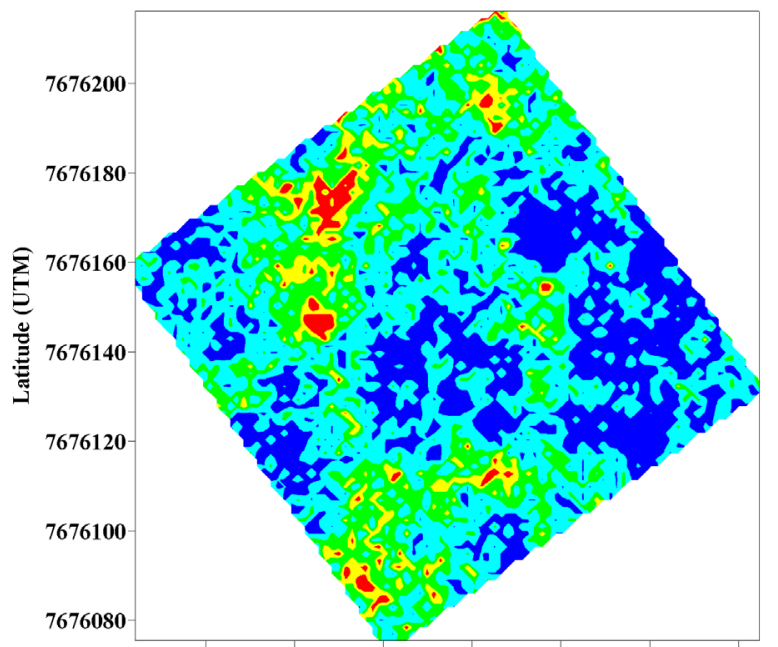

$\begin{array}{llllllll}699400 & 699420 & 699440 & 699460 & 699480 & 699500 & 699520\end{array}$

E. Krigagem Ordinária

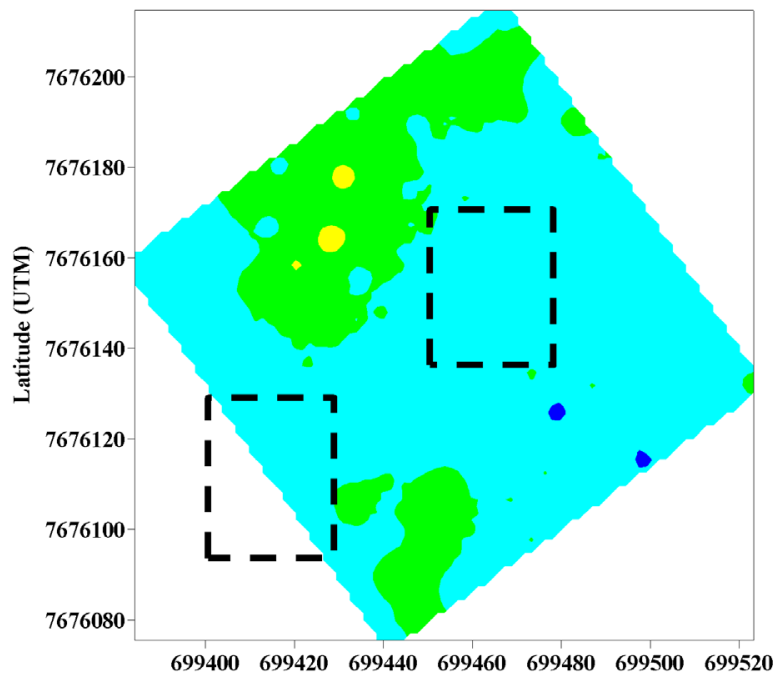

Longitude (UTM)
B. SSG $37^{\mathrm{a}}$

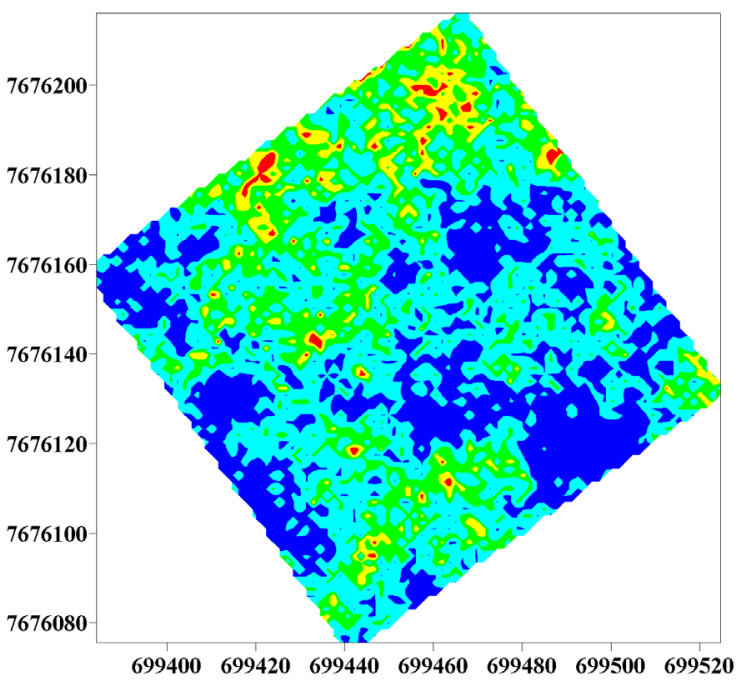

D. SSG $200^{\mathrm{a}}$

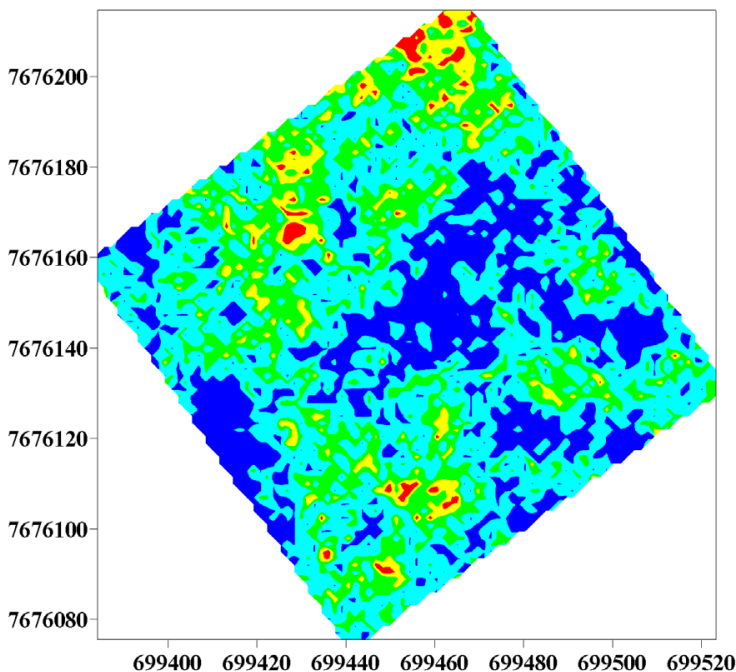

F. E-Type

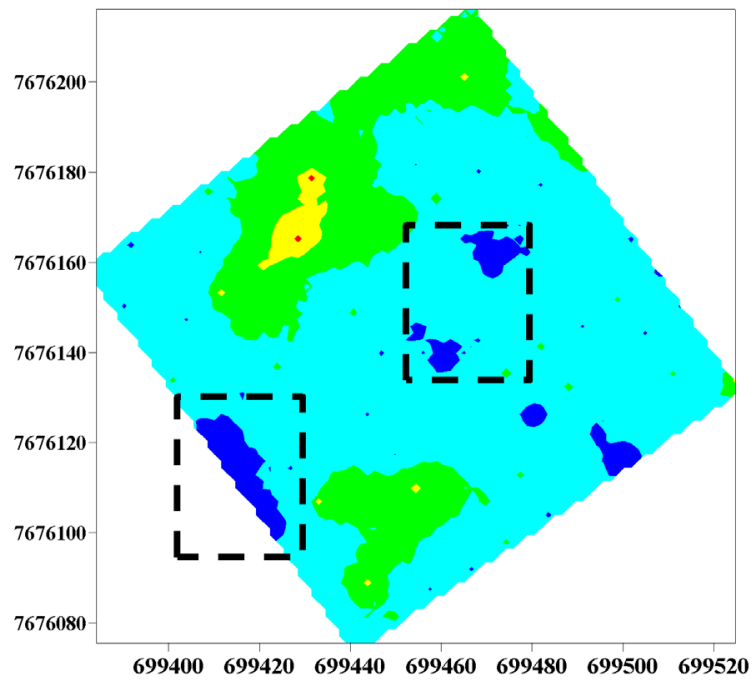
Longitude (UTM)

\section{$\begin{array}{lllll}14 & 24 & 34 & 44 & 54\end{array}$}

Figura 4. Mapas do diâmetro médio do cristal da goethita $(\mathrm{nm})$ : realizações sorteadas da SSG (SSG1ª; SSG37aa; SSG128ª e SSG200ª), média das 200 realizações (E-type) e krigagem ordinária 
conforme retângulo pontilhado Figuras 4E e 4F. Este fato pode ocasionar decisões errôneas no uso desses mapas gerados pela KO pois nesses locais se superestima o tamanho do cristal informando indiretamente que a área da superfície específica (ASE) será menor podendo ocasionar erros nas adubações fosfatadas em que nesses locais mais fósforo será fixado visto que, na realidade, possui menor DMC e indiretamente maior a ASE (Rolim Neto et al., 2004), o que atribui maior capacidade de adsorção de fósforo no solo.

Do ponto de vista prático, não se recomenda o uso dos mapas das realizações individuais da SSG pois apresentam limitações relacionadas principalmente ao excesso de detalhamento do mapa produzido (Soares, 2006). No entanto, são recomendados os mapas das estimativas E-Type conforme constatado por Silva Júnior et al. (2012a) no mapeamento de óxidos de ferro.

Quanto menor a cristalinidade da Gt maior é o seu valor da superfície específica; a baixa cristalinidade desse óxido de ferro está associada à maior adsorção de fosfato (Rolim Neto et al., 2004). Notam-se, visualmente, retângulo pontilhado nas Figuras $4 \mathrm{E}, \mathrm{F}$ e o efeito negativo da suavização da $\mathrm{KO}$, superestimando áreas com menores valores de DMC e, de maneira indireta, subestima o potencial de adsorção de fósforo de vez que não apresenta locais específicos com valores extremos (mínimos e máximos) do DMC que podem ser considerados importantes no manejo do solo visando reduzir a adsorção de P.

Este fato pode levar a decisões errôneas, caso for necessário fazer adubações fosfatadas baseadas nesses mapas de KO. Efeitos semelhantes à suavização da $\mathrm{KO}$ foram relatados por (Lookman et al., 1995). Isto deixa explícito que estimativas realizadas por meio da $\mathrm{KO}$ necessitam de um fator de correção como forma de amenizar os efeitos da suavização verificada no uso desta técnica (Santos et al., 2011).

Os modelos das realizações da SSG estão mais consistentes uma vez que se encontram mais próximos do modelo de semivariograma do DMC da Gt enquanto o modelo da $\mathrm{KO}$ não tem o mesmo desempenho em reproduzir o modelo do semivariograma de referência, apresentando o maior erro em relação ao do modelo de semivariograma do DMC da Gt. Esses resultados são semelhantes aos reportados por Delbari et al. (2009).

Desta forma, as estimativas E-Type pela SSG mostram seu potencial na caracterização espacial do DMC da Gt, na definição de zonas "homogêneas" de manejo semelhante a KO, embora com maior precisão, sendo considerado o método geoestatístico mais indicado em estudos de variabilidade espacial dos óxidos de ferro quando se prevê o realismo do fenômeno (Silva Júnior et al., 2012a).

Os valores de $\varepsilon_{\mathrm{y}}$ foram crescentes na seguinte ordem: as realizações individuais da SSG128 ${ }^{\mathrm{a}}, \mathrm{SSG} 200^{\mathrm{a}}, \mathrm{SSG}^{\mathrm{a}}, \mathrm{SSG} 37^{\mathrm{a}}$, E-Type e KO indicando que nesta ordem ocorre diminuição da acurácia na reprodução do semivariograma do conjunto amostral (Figura 5).

Como já referenciado, é importante utilizar a média das realizações (E-type); em relação à $\mathrm{KO}$, obteve o maior valor de $\varepsilon_{\mathrm{y}}$ que foi 5,99 enquanto o valor de $\varepsilon_{\mathrm{y}}$ para E-Type de 4,55 confirma um aumento de acurácia e confiança do mapa médio das 200 realizações em relação a KO. Este resultado é mais uma implicação que pode ser atribuída ao efeito de

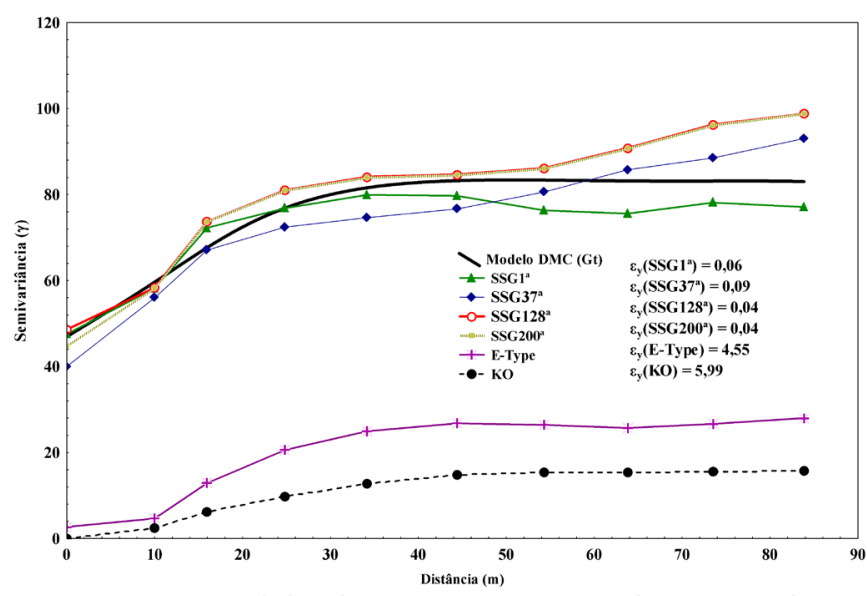

Figura 5. Modelo de semivariograma do $\mathrm{DMC}$, das realizações sorteadas $\mathrm{n}^{0} 1\left(\mathrm{SSG} 1^{\mathrm{a}}\right), \mathrm{n}^{\mathrm{o}} 37\left(\mathrm{SSG} 37^{\mathrm{a}}\right), \mathrm{n}^{\mathrm{o}}$ $128\left(\right.$ SSG128 $\left.^{\mathrm{a}}\right)$ e $\mathrm{n}^{\mathrm{o}} 200\left(\mathrm{SSG}^{200^{\mathrm{a}}}\right.$ ), média de 200 realizações (E-Type), krigagem ordinária $(\mathrm{KO})$ e erro na reprodução do semivariograma $\left(\varepsilon_{\mathrm{y}}\right)$

suavização da estimativa da $\mathrm{KO}$ visto que houve subestimação da variabilidade em pequenas distâncias, além de um ajuste do semivariograma ao modelo gaussiano, característico de variáveis com distribuição suave no espaço.

Apesar da formação dos solos não ocorrer de maneira aleatória (estocástica), a Figura 5 mostra um indicativo de que seus atributos mineralógicos podem ter este comportamento sendo recomendado utilizar a simulação estocástica SSG para a caracterização espacial confirmando a hipótese de Yamamoto (2005) e Santos et al. (2011) de que se deve fazer uma correção do efeito de suavização da krigagem ordinária para obter mapas de variabilidade espacial mais próximo à variabilidade capturada na modelagem do semivariograma e concordando com o trabalho de Delbari et al. (2009) e Silva Júnior et al. (2012a), em que é preferível o uso de mapas das estimativas E-Type da SSG ao invés da KO quando se deseja maior acurácia no mapeamento.

Deste modo, é essencial testar outros algoritmos e técnicas que incorporem qualidade das informações geradas pela inferência espacial, conforme já reportado por Herbst et al. (2010) e Siqueira et al. (2010). Esta afirmação foi comprovada por Silva Júnior et al. (2012b) em que a combinação da geoestatística com análise, juntamente com modelos de paisagem, é eficiente na caracterização espacial de atributos dos solos.

É importante destacar que na escolha dos métodos de inferência espacial do DMC da Gt e conhecer os possíveis erros e desvantagens deles é tão importante quanto apenas produzir mapas para aplicação localizada de adubos fosfatados.

Assim sendo, os usuários da ferramenta geoestatística e/ou os tomadores de decisão devem estar cientes da necessidade de avaliar a qualidade da informação gerada pelos modelos dos semivariograma pois no mapeamento do DMC da Gt não é suficiente a caracterização da variabilidade espacial média, visto que as pequenas diferenças na sua área específica mais elevada (menor tamanho do cristalino) assumem papel fundamental na fase rápida e lenta de adsorção, com a presença de maior número de grupamentos $\mathrm{OH}^{-}$aumentando as reações da superfície desse mineral com o fósforo (P) (Rolim Neto et al., 2004). 


\section{Conclusões}

1. A simulação sequencial gaussiana reproduz e representa com maior confiança nos resultados da variabilidade espacial do diâmetro médio do cristal da goethita, sendo recomendado utilizar mapas gerados pela estimativa E-type.

2. A krigagem ordinária reproduz apenas a variabilidade média da amostra do diâmetro médio do cristal da goethita, sendo recomendado usar técnicas de correção do efeito da suavização.

\section{Agradecimentos}

Os autores agradecem à CAPES, pela concessão da bolsa de estudo.

\section{Literatura Citada}

Bourennane, H.; Douayb, F.; Sterckemanc, T.; Villanneaud, E.; Ciesielskie, H.; Kinga, D.; Baizea, D. Mapping of anthropogenic trace elements inputs in agricultural topsoil from Northern France using enrichment factors. Geoderma, v.157, p.165-174, 2010.

Bourennane, H.; King, D.; Couturier, A.; Nicoullaud, B.; Mary, B.; Richard, G. Uncertainty assessment of soil water content spatial patterns using geosetatistical simulations: An empirical comparison of a simulation accounting for secondary information. Ecological Modelling, v.205, p.323-335, 2007.

Burgess, T. M.; Webster, R. Optimal interpolation and isarithmic mapping of soil properties. I. The variogram and punctual kriging. Journal Soil Science, v.31, p.315-331, 1980.

Camargo, L. A.; Marques Júnior, J.; Pereira, G. T.; Horvat, R. A. Variabilidade espacial de atributos mineralógicos de um latossolo sob diferentes formas de relevo. I-Mineralogia da fração argila. Revista Brasileira de Ciência do Solo, v.32, p.2269-2277, 2008.

Cambardella, C. A.; Moorman, T. B.; Novak, J. M.; Parkin, T. B.; Karlen, D. L.; Turco, R. F. Konopka, A. E. Field-scale variability of soil properties in central Iowa soils. Soil Science Society of America Journal, v.58, p.1501-1511, 1994.

Delbari, M.; Afrasiab, P.; Loiskandl, W. Using sequential gaussian simulation to assess the field-scale spatial uncertainty of soil water content. Catena, v.79, p.163-169, 2009.

Deutsch, C. V.; Journel, A. G. GSLIB: Geostatistical software library: and user's guide, 2.ed. New York: Oxford University Press, 1998. 369p.

EMBRAPA - Empresa Brasileira de Pesquisa Agropecuária. Centro Nacional de Pesquisa de Solos. Sistema brasileiro de classificação de solos. Rio de Janeiro: Embrapa CNPS. 2006. 306p.

Goovaerts, P. Estimation or simulation of soil properties? An optimization problem with conflicting criteria. Geoderma, v.97, p.165-186, 2000.

Herbst, M.; Prolingheuer, N.; Graf, A.; Huisman, J. A.; Weihermüller, L.; Vanderborght, J.; Vereecken, H. Multivariate conditional stochastic simulation of soil heterotrophic respiration at plot scale. Geoderma, v.160, p.74-82, 2010.
Isaaks, E. H.; Srivastava, R. M. Applied geostatistics. New York: Oxford University Press, 1989. 561p.

Journel, A. G. Fundamentals of geostatistics in five lessons. Washington: Americam Geographysical Union, 1989. p.673-687.

Kämpf, N.; Schwertmann, U. Goethite and hematite in a climosequênce in Southern Brazil and their application in classification of kaolinitic Soils. Geoderma, v.29, p.2739,1982 .

Lookman, R.; Vandeweert, N.; Merckx, R.; Vlasaak, K. Geostatistical assessment of the regional distribution of phosphate sorption capacity parameters (feox and alox) in northern Belgium. Geoderma, v.66, p.285-296, 1995.

Matheron, G. Principles of geostatistics. Economic Geology, v.58, p. 1246-1266, 1963.

McBratney, A. B.; Santos, M. L. M.; Minasny, B. On digital soil mapping. Geoderma, v.117, p.3-52, 2003.

Montanari, R.; Marques Júnior, J.; Campos, M. C. C.; Souza, Z. M.; Camargo, L. A. Caracterização mineralógica de Latossolos em diferentes feições do relevo na região de Jaboticabal, SP. Revista Ciência Agronômica, v.41, p.191199, 2010.

Oliveira Júnior, J. C.; Souza, L. C. P.; Melo, V. F.; Rocha, H. O. Variabilidade espacial de atributos mineralógicos de solos da formação guabirotuba, Curitiba (PR). Revista Brasileira de Ciência do Solo, v.35, p.1481-1490, 2011.

Ortiz, J. O.; Felgueiras, C. A.; Druck, S.; Monteiro, A. M. V. Modelagem de fertilidade do solo por simulação estocástica com tratamento de incertezas. Pesquisa Agropecuária Brasileira, v.39, p.379-389, 2004.

Rezaee, H.; Asghari, O.; Yamamoto, J. K. On the reduction of the ordinary kriging smoothing effect. Journal of Mining \& Environment, v.2, p.102-117, 2011.

Rolim Neto, F. C.; Schaefer, C. E. G. R.; Costa, L. M.; Corrêa, M. M.; Fernandes Filho, E. I.; Ibraimo, M. M. Adsorção de fósforo, superfície específica e atributos mineralógicos em solos desenvolvidos de rochas vulcânicas do Alto Paranaíba (MG). Revista Brasileira de Ciência do Solo, v.28, p.953964, 2004.

Santos, P. C.; Santana, A. C.; Barros, P. L. C.; Queiroz, J. C. B.; Vieira, T. O. O emprego da geoestatística na determinação do tamanho "ótimo" de amostras aleatórias com vistas à obtenção de estimativas dos volumes dos fustes de espécies florestais em Paragominas, estado do Pará. Acta Amazônica, v.41, p.213-222, 2011.

Silva Júnior, J. F.; Marques Jr, J.; Camargo, L. A.; Teixeira, D. D. T.; Panosso, A. R.; Pereira, G. T. Simulação geoestatística na caracterização espacial de óxidos de ferro em diferentes pedoformas. Revista Brasileira de Ciência do Solo, v.36, p.1690-1703, 2012a.

Silva Júnior, J. F.; Siqueira, D. S.; Marques Jr, J. Pereira, G. T. Classificação numérica e modelo digital de elevação na caracterização espacial de atributos dos solos. Revista Brasileira de Engenharia Agrícola e Ambiental, v.16, p.415424, 2012b.

Siqueira, D. S.; Marques Júnior, J.; Pereira, G. T. The use of landforms to predict the variability of soil and orange attributes. Geoderma, v.155, p.55-66, 2010. 
Soares, A. Geoestatística para a ciência da terra e do ambiente. 2. ed. Lisboa: IFT Press, 2006. 214p.

Teixeira, D. D. B.; Panosso, A. R.; Cerri, C. E. P.; Pereira, G. T.; La Scala, N. Soil CO2 emission estimated by different interpolation techniques. Plant and Soil, v.345, p.187-194, 2011.

Warrick, A. W.; Nielsen, D. R. Spatial variability of soil physical properties in the field. In: Hillel, D., ed. Applications of soil physics. New York, Academic Press, 1980. p.319-344.
Yamamoto, J. K. Correcting the smoothing effect of ordinary kriging estimates. Mathematical Geology, v.37, p.69-94, 2005.

Zanão Júnior, L. A.; Lana, R. M. Q.; Zanão, M. P. C.; Guimarães, E. C. Variabilidade espacial de atributos químicos em diferentes profundidades em um Latossolo em sistema de plantio direto. Revista Ceres, v.57, p.429438, 2010. 